ARTICLE

\title{
Ladder-like energy-relaying exciplex enables 100\% internal quantum efficiency of white TADF-based diodes in a single emissive layer
}

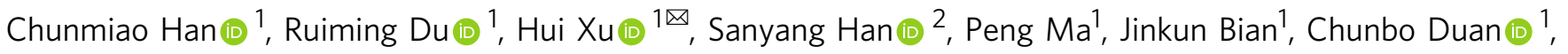

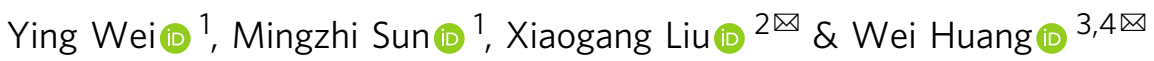

Development of white organic light-emitting diodes based on purely thermally activated delayed fluorescence with a single-emissive-layer configuration has been a formidable challenge. Here, we report the rational design of a donor-acceptor energy-relaying exciplex and its utility in fabricating single-emissive-layer, thermally activated delayed fluorescencebased white organic light-emitting diodes that exhibit 100\% internal quantum efficiency, $108.2 \mathrm{~lm} \mathrm{~W} \mathrm{~W}^{-1}$ power efficiency, and $32.7 \%$ external quantum efficiency. This strategy enables thin-film fabrication of an $8 \mathrm{~cm} \times 8 \mathrm{~cm}$ thermally activated delayed fluorescence white organic light-emitting diodes (10 inch ${ }^{2}$ ) prototype with $82.7 \mathrm{~lm} \mathrm{~W} \mathrm{~m}^{-1}$ power efficiency and $25.0 \%$ external quantum efficiency. Introduction of a phosphine oxide-based acceptor with a steric group to the exciplex limits donor-acceptor triplet coupling, providing dual levels of high-lying and low-lying triplet energy. Transient spectroscopic characterizations confirm that a ladder-like energy relaying occurs from the high-lying triplet level of the exciplex to a blue emitter, then to the low-lying triplet level of the phosphine oxide acceptor, and ultimately to the yellow emitter. Our results demonstrate the broad applicability of energy relaying in multicomponent systems for exciton harvesting, providing opportunities for the development of third-generation white organic light-emitting diode light sources.

\footnotetext{
${ }^{1}$ Key Laboratory of Functional Inorganic Material Chemistry (Ministry of Education) \& School of Chemistry and Material Science, Heilongjiang University, Harbin, PR China. ${ }^{2}$ Department of Chemistry, National University of Singapore, Singapore, Singapore. ${ }^{3}$ Key Laboratory of Flexible Electronics \& Institute of Advanced Materials, Nanjing Tech University, Nanjing, China. ${ }^{4}$ Frontiers Science Center for Flexible Electronics (FSCFE) \& Shaanxi Institute of Flexible Electronics (SIFE), Northwestern Polytechnical University (NPU),Xi'an, China. ${ }^{\circledR}$ email: hxu@hlju.edu.cn; chmlx@nus.edu.sg; iamwhuang@nwpu.edu.cn
} 
C onsiderable effort has recently been devoted to developing high-efficiency, white organic light-emitting diodes (WOLEDs) with compact design and large-area processing capability ${ }^{1-3}$. Thermally activated delayed fluorescence (TADF), based on purely organic emitters, enables theoretical $100 \%$ internal quantum efficiency for both singlet and triplet exciton harvesting ${ }^{4-6}$. A single-emissive layer (EML) design, comprising blue/yellow emitters or red/green/blue emitters, can simplify WOLED device structure effectively and can meet the demands of large-scale production, quality control, and low $\operatorname{cost}^{7-9}$. However, competition in exciton confinement between various color emitters makes it challenging to control emission color and device efficiency synchronously (Fig. 1) ${ }^{10,11}$. As an additional constraint, charge-transfer excited states of TADF dyes are highly sensitive to host-dopant interactions ${ }^{12-14}$ and interfacial effects ${ }^{15}$. Indeed, there are few reports of high-efficiency, purely TADF-based WOLEDs, among which multiple emissive layers are required to modify exciton allocation by spatially separating two or three host-dopant systems of different emission color ${ }^{16-18}$.

High-efficiency WOLEDs with single-EML layout requires precise optimization of optical transition and energy transfer (ET) in a multi-emitter-doped host ${ }^{19}$. However, the narrow singlettriplet splitting energy $\left(\Delta \mathrm{E}_{\mathrm{ST}}\right)$ of TADF emitters limits energylevel regulation. The singlet-triplet energy gap between the blue and yellow TADF emitters, e.g., bis[4-(9,9-dimethyl-9,10-dihydroacridine)phenyl]sulfone (DMAC-DPS, $\mathrm{S}_{1} / \mathrm{T}_{1} \approx 2.8 \mathrm{eV}$ ) ${ }^{20}$ and 2,3,5,6-tetrakis(3,6-di-t-butylcarbazol-9-yl)-1,4-dicyanobenzene (4CzTPNBu, $\left.\mathrm{S}_{1} / \mathrm{T}_{1} \approx 2.2 \mathrm{eV}\right)^{4}$, is approximately $0.6 \mathrm{eV}$, leading to nonradiative deactivation of excitons during energy transfer ${ }^{21-23}$.

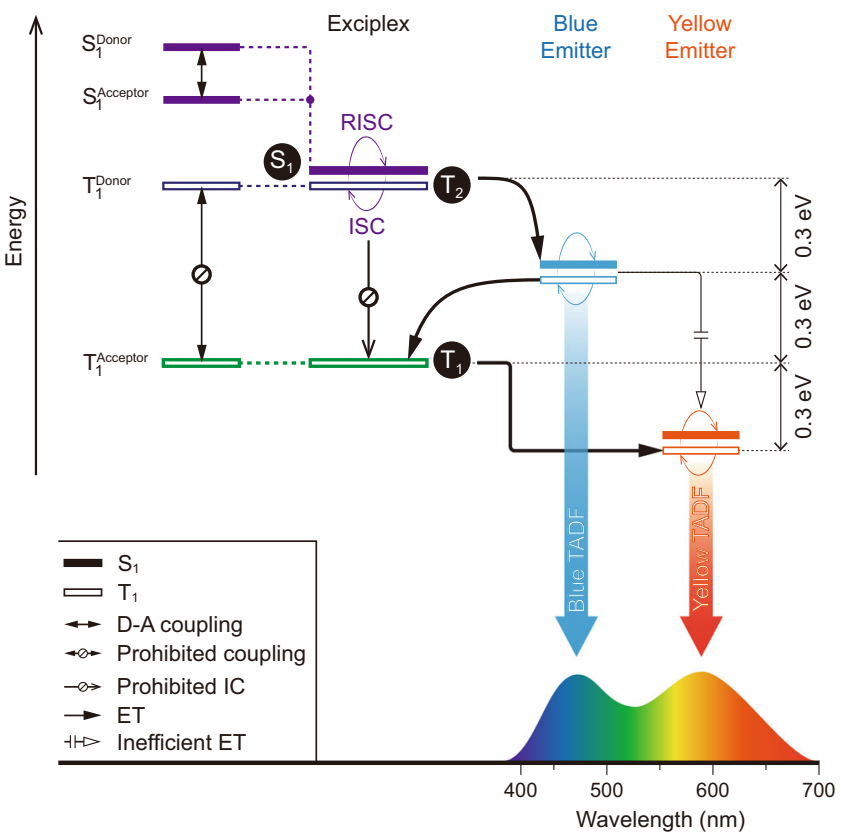

Fig. 1 Proposed energy-relaying mechanism. Exciton allocation in singleemissive-layer, TADF WOLEDs comprising a D-A exciplex host, a blue emitter, and a yellow emitter. Inefficient energy transfer occurs from the exciplex host to the blue emitter and subsequently to the yellow emitter, due to a large energy gap between the blue and yellow emitters $(\sim 0.6 \mathrm{eV})$. The proposed exciplex, based on a high triplet donor and a low triplet acceptor, generates a large triplet gap between a high-lying $\mathbf{T}_{\mathbf{2}}$ and a lowlying $\mathbf{T}_{\mathbf{1}}$. This design enables efficient ladder-like ( $0.3 \mathrm{eV}$ ladder spacing) triplet energy transfer from $\mathbf{T}_{\mathbf{2}} \rightarrow$ blue emitter $\rightarrow \mathbf{T}_{\mathbf{1}} \rightarrow$ yellow emitter. ET, IC, ISC, and RISC refer to energy transfer, internal conversion, intersystem crossing, and reverse ISC, respectively.
Presently, the performance of single-EML, TADF-based WOLEDs is not comparable to that of their phosphorescent counterparts $^{24,25}$. We reason that a host matrix with dual levels of high-lying and low-lying triplet energy, which match the energy levels of blue and yellow TADF emitters, may improve performance ${ }^{23,26}$. In contrast, single-molecule-based hosts cannot offer two triplet excited states, due to fast internal conversion to the lowest triplet state $\left(\mathrm{T}_{1}\right)$. Therefore, an exciplex host based on a bimolecular donor-acceptor (D-A) system is likely to provide two different triplet states upon appropriate D-A coupling 27,28 . In principle, a common exciplex comprises a donor and an acceptor with comparable excited energy levels and strong D-A coupling, lowering $S_{1}$ and $T_{1}$ levels of the exciplex below those of the donor and the acceptor. In contrast, an exciplex design featuring a large triplet energy gap between the donor and the acceptor limits donor-acceptor triplet coupling (Fig. 1) ${ }^{29}$. This leads to dual triplet levels of the exciplex with an energy difference of $0.6 \mathrm{eV}$. Therefore, a facile triplet energy transfer process may occur, namely the $\mathrm{S}_{1} / \mathrm{T}_{2}$ of the exciplex host $\rightarrow$ the $\mathrm{S}_{1} / \mathrm{T}_{1}$ of blue emitter $\rightarrow$ the $T_{1}$ of the exciplex host $\rightarrow$ the $T_{1}$ of yellow emitter. As an added benefit, the exciplex host with near-zero $\Delta \mathrm{E}_{\mathrm{ST}}$ can provide additional triplet-singlet conversion and enhance singlet-exciton utilization in the blue-TADF emitter.

As a proof of concept, we designed and synthesized two exciplex hosts, mCP:pDPBITPO and mCP:DpPBITPO, with $\mathrm{T}_{2}$ and $\mathrm{T}_{1}$ energy levels of 3.0 and $2.5 \mathrm{eV}$, based on a high-tripletenergy donor, 1,3-bis (carbazol-9-yl)benzene ( $\left.\mathrm{mCP}, \mathrm{T}_{1}=3.0 \mathrm{eV}\right)$ and low-triplet-energy phosphine oxide (PO) acceptors (5-(2(4-(diphenylphosphoryl)phenyl)-benzimidazol-1-yl)-1,3-phenylene)bis(diphenylphosphine oxide) (pDPBITPO) and (5-(1(4-(diphenylphosphoryl)phenyl)-benzoimidazol-2-yl)-1,3-phenylene)bis(diphenylphosphine oxide) (DpPBITPO) $\left(\mathrm{T}_{1}=2.5 \mathrm{eV}\right.$ as the average of DMAC-DPS and $4 \mathrm{CzTPNBu}$ ) (Fig. 2a). Our experimental results show that these two exciplexes promote energy transfer relaying in their DMAC-DPS and $4 \mathrm{CzTPNBu}-$ codoped films. A single-EML design based on mCP:pDPBITPO and mCP:DpPBITPO exciplex yielded TADF WOLEDs with a maximum external quantum efficiency $\left(\eta_{\mathrm{EQE}}\right)$ of $32.7 \%$ and a maximum power efficiency of $108.2 \mathrm{~lm} \mathrm{~W}^{-1}$, comparable to that of fluorescent lamps.

\section{Results}

Exciplex host design. The key molecular design of pDPBITPO and DpPBITPO involves electron-accepting phenylbenzimidazole encapsulated by three diphenylphosphine oxide (DPPO) moieties to provide suitable frontier molecular orbital energy levels and to reduce intermolecular charge-transfer interactions with $\mathrm{mCP}^{27}$ (Fig. 2a). Furthermore, the single-crystal structure of DpPBITPO reveals a twisted configuration of the phenylbenzimidazole group due to steric hindrance of the DPPO moiety (Supplementary Fig. 1). This leads to improved triplet-state structural relaxation, reduced $\mathrm{T}_{1}$ energy level, and enlarged $\Delta \mathrm{E}_{\mathrm{ST}}$. Density functional theory (DFT) simulations show locally excited $\mathrm{T}_{1}$ states of $\mathrm{mCP}$, pDPBITPO and DpPBITPO (Supplementary Fig. 2 and Supplementary Table 1). $\mathrm{T}_{1}$ energy gaps between the donor and the two acceptors reach $\sim 0.8 \mathrm{eV}$, which is adequate to restrain $\mathrm{D}-\mathrm{A}$ triplet exciton interactions. As a result of suppressed triplet $\mathrm{D}-\mathrm{A}$ electronic coupling, high-lying triplet $\left(\mathrm{T}_{2}\right)$ and $\mathrm{T}_{1}$ states of the exciplexes are localized at $\mathrm{mCP}$ and $\mathrm{PO}$ acceptors, respectively. Moreover, the energy gaps between the highest occupied (HOMO) and the lowest unoccupied molecular orbital (LUMO) energy levels of $\mathrm{mCP}$ and PO acceptors reach $\sim 0.6 \mathrm{eV}$ (Fig. $2 \mathrm{~b}$ and Supplementary Figs. 3). This suggests strong driving forces of $\mathrm{D}-\mathrm{A}$ charge transfer upon excitation, giving rise to chargetransfer $S_{1}$ states. Effective spin-orbital coupling between the 
a

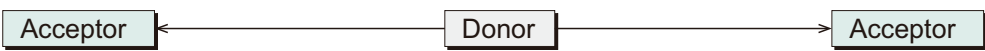

E

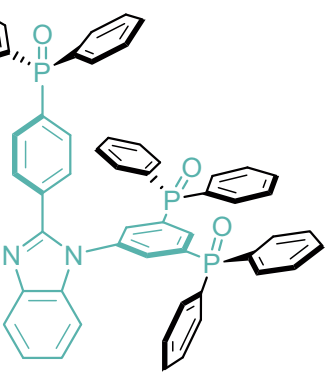

pDPBITPO

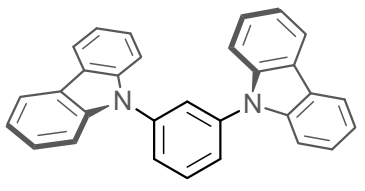

$\mathrm{mCP}$

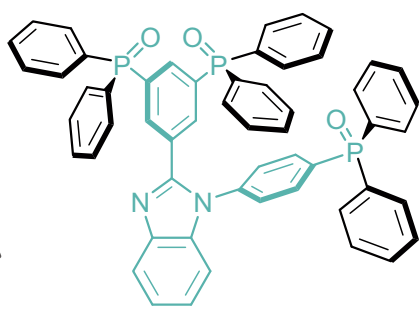

DPPBITPO

$\uparrow$ mCP:DpPBITPO

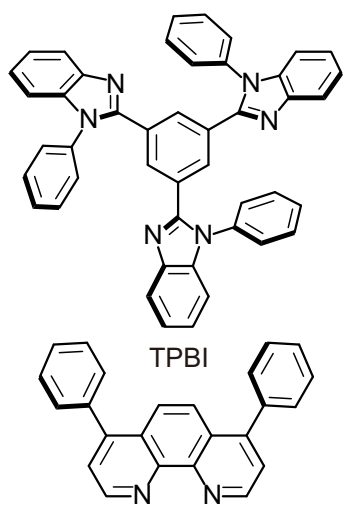

Bphen b

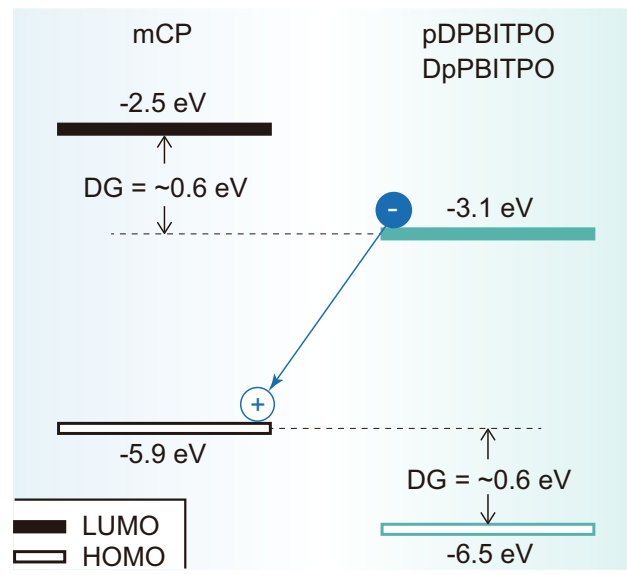

d

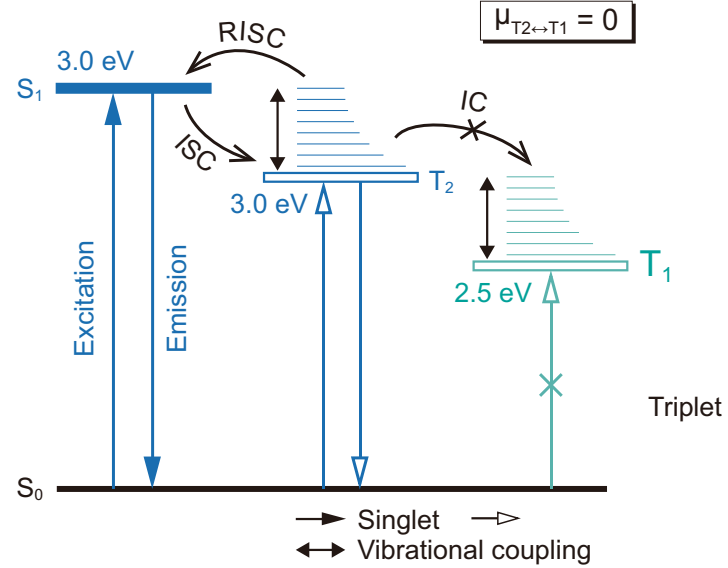

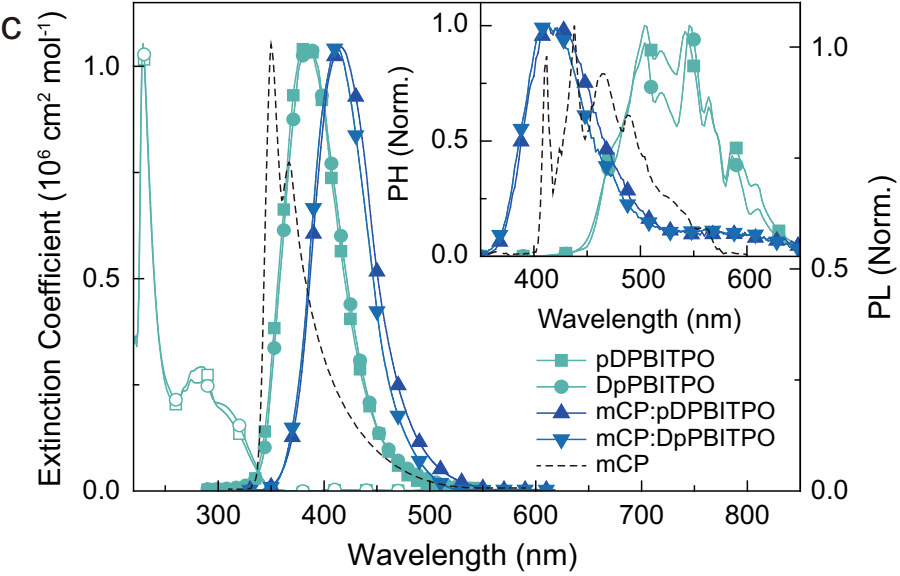

e

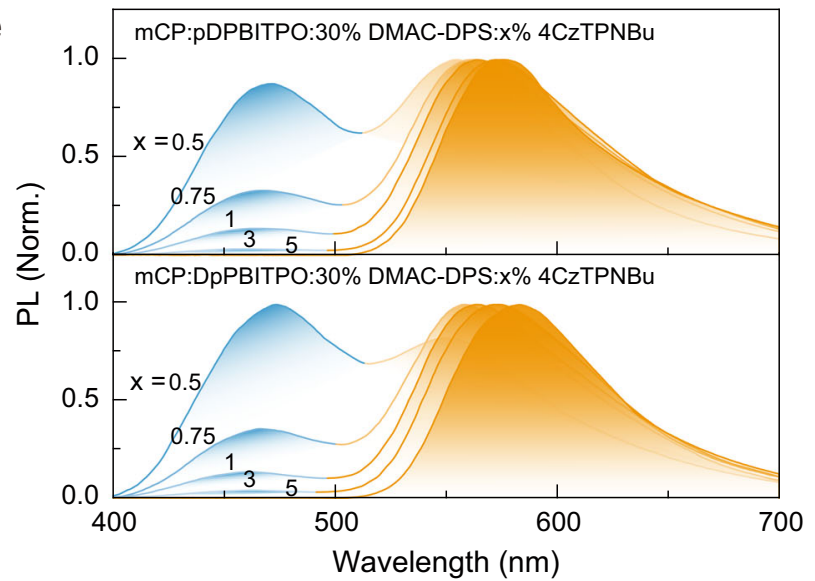

Fig. 2 Structure and energy transition characteristics of the newly developed exciplexes. a Molecular structures of one donor and two acceptors used to form exciplexes mCP:pDPBITPO and mCP:DpPBITPO. Two conventional TPBI and BPhen acceptors were used in device fabrication for comparison. b Frontier molecular orbital (FMO) energy levels of $\mathrm{mCP}$ and PO acceptors. Energy gaps of the highest occupied (HOMO) and the lowest unoccupied molecular orbitals (LUMO) between $\mathrm{mCP}$ and $\mathrm{PO}$ acceptors reach to $0.6 \mathrm{eV}$, corresponding to the driving force of donor-acceptor electronic coupling $(\Delta G)$. c Electronic absorption of pDPBITPO and DpPBITPO (hollow dots) and steady-state photoluminescence (PL) and time-resolved phosphorescence $(\mathrm{PH})$ spectra of $\mathrm{mCP}$ (dash lines), pDPBITPO, DpPBITPO, mCP:pDPBITPO and mCP:DpPBITPO. Phosphorescence spectra were recorded in the time range of 100-200 $\mu$ s after excitation. $\mathbf{d}$ Jablonski energy level diagram of mCP:PO acceptors. The near-zero energy gap between the $\mathrm{S}_{1}$ and the mCP-induced $\mathrm{T}_{2}$ of the exciplex facilitates the RISC through electron and vibrational couplings. Separated $T_{2}$ and $T_{1}$ locations on $m C P$ and $P O$ acceptors restrain the $T_{2} \rightarrow T_{1}$ transition through IC. e Steady-state PL spectra of mCP:pDPBITPO and mCP:DpPBITPO films doped with DMAC-DPS and 4CzTPNBu at different ratios.

locally excited triplet state and the charge-transfer $S_{1}$ state substantially facilitates reverse intersystem crossing (RISC), as evident in the cases of mCP:pDPBITPO and mCP:DpPBITPO $\left(\right.$ ref. $\left.{ }^{30}\right)$. Moreover, PO acceptors are superior to conventional low-triplet-energy acceptors with exposed $\mathrm{T}_{1}$ states, such as TPBI $\left(\mathrm{T}_{1}=2.7 \mathrm{eV}\right)$ and BPhen $\left(\mathrm{T}_{1}=2.6 \mathrm{eV}\right)$, due to prevention of collision-induced quenching at the $\mathrm{T}_{1}$ state by peripheral DPPO groups.

Estimated by $0 \rightarrow 0$ transitions in emission spectra, the $T_{1}$ energy levels $(2.48 \mathrm{eV})$ of pDPBITPO and DpPBITPO match well with $4 \mathrm{CzTPNBu}(2.2 \mathrm{eV})$, while featuring triplet gaps that are $0.54 \mathrm{eV}$ lower than $\mathrm{mCP}\left(\mathrm{T}_{1}=3.02 \mathrm{eV}\right)$. Meanwhile, the $\mathrm{S}_{1}$ 
energy levels of the $\mathrm{PO}$ acceptors are $3.2 \mathrm{eV}$, similar to $\mathrm{mCP}$ $\left(\mathrm{S}_{1}=3.5 \mathrm{eV}\right)$. The deep-blue emissions from co-evaporated $\mathrm{mCP}$ : PO acceptor films exhibit a bathochromic shift of $30 \mathrm{~nm}$, corresponding to Gibbs free energy for charge separation beyond $0.2 \mathrm{eV}$, indicating exciplex formation through $\mathrm{D}-\mathrm{A}$ electronic coupling (Fig. 2c; Supplementary Figs. 4 and 5; Supplementary Table 2) (ref. ${ }^{29}$ ). The D-A vibrational coupling leads to structureless phosphorescence spectra of mCP:pDPBITPO and $\mathrm{mCP}: \mathrm{DpPBITPO}$. The main peaks at $415 \mathrm{~nm}$ correspond to the $0 \rightarrow 0$ triplet transition of $\mathrm{mCP}$, namely the $\mathrm{mCP}$-centered $\mathrm{T}_{2} \rightarrow$ $\mathrm{S}_{0}$ transitions. Therefore, the $\mathrm{T}_{2}$ levels of the exciplexes $(\sim 3.0 \mathrm{eV})$ support efficient energy transfer to DMAC-DPS. Furthermore, the tails of the phosphorescence spectra from 500 to $650 \mathrm{~nm}$ overlap with those of the PO acceptors, which can be ascribed to either the $\mathrm{T}_{1} \rightarrow \mathrm{S}_{0}$ transitions of the exciplexes or to triplet leakage to the $T_{1}$ state of acceptors ${ }^{29}$. Compared to $T_{1}$ states located at the PO acceptor, $\mathrm{mCP}$-induced $\mathrm{T}_{2}$ states are closer to $\mathrm{S}_{1}$ states, supporting RISC transitions. In the case of weak D-A triplet exciton interactions and $\mathrm{mCP}$-centralized HOMOs, these $\mathrm{T}_{2}$ states dominate the triplet population. Furthermore, D-A vibrational coupling restrains vibrational perturbation of potential energy surfaces and further alleviates internal conversion from the $\mathrm{T}_{2}$ to $\mathrm{T}_{1}$ states (Fig. $2 \mathrm{~d}$ ), closely resembling rigid, intramolecular charge-transfer systems ${ }^{31}$. Despite the decreased photoluminescence quantum yields $\left(\phi_{\mathrm{PL}}\right)$ of the exciplexes $(<15 \%)$ (Supplementary Table 2 ), the $\mathrm{PO}$ acceptor-induced $\mathrm{T}_{1}$ states can serve as intermediate states to promote triplet energy transfer to $4 \mathrm{CzTPNBu}$.

Photophysical investigation on the energy-relaying process. The DMAC-DPS (30 wt $\%$ ) and 4CzTPNBu (5 wt\%) singly doped sky-blue and yellow exciplex films $(100 \mathrm{~nm})$ showed $\sim 30 \%$ and $\sim 65 \%$ increased $\phi_{\mathrm{PL}}$, respectively (Fig. 2e; Supplementary Table 2). These values are still far below the intrinsic values of the dopants ( 95\%), due to either triplet leakage to the $\mathrm{T}_{1}$ of the PO acceptors (for DMAC-DPS) or the large energy gap-induced inefficient energy transfer (for 4CzTPNBu). For the doubly doped films of mCP:PO acceptor:30\% DMAC-DPS:x\% 4CzTPNBu (x= $0.5-5)$, they all achieved $\phi_{\mathrm{PL}}$ values above $90 \%$, approximately the sum of the singly doped films.

The weakly populated bands at $500 \mathrm{~nm}$, observed in the time-resolved contours of $\mathrm{mCP}: \mathrm{PO}$ acceptor exciplexes due to the phosphorescence from the PO acceptors, could be dramatically enhanced upon doping of $30 \%$ DMAC-DPS (Fig. 3a). The triplet energy was transferred from DMACDPS to PO acceptors at about $70 \mathrm{~ns}$, followed by the appearance of dominant phosphorescence bands at $150 \mathrm{~ns}$. For mCP:PO acceptor:5\% 4CzTPNBu, we only observed blue bands from the exciplexes within $50 \mathrm{~ns}$. The absence of phosphorescence bands indicates that PO acceptors do not participate in triplet energy transfer to $4 \mathrm{CzTPNBu}$ due to forbidden $T_{2} \rightarrow T_{1}$ transitions of the exciplexes. The timeresolved emission spectra (TRES) of $\mathrm{mCP}: \mathrm{PO}$ acceptor: $30 \%$ DMAC-DPS:0.5\% 4CzTPNBu films were measured to examine the exact energy transfer process (Fig. $3 \mathrm{~b}$ ). In the time range of $100-500 \mathrm{~ns}$, along with the decrease of the bands assigned to DMAC-DPS $(\sim 470 \mathrm{~nm})$, the bands that peaked at $\sim 500 \mathrm{~nm}$ gradually became dominant, which could be attributed to phosphorescence from the PO acceptors. After the coexistence of the phosphorescent and yellow bands during this time range, the yellow bands remained while the phosphorescence bands vanished. Furthermore, despite the low $4 \mathrm{CzTPNBu}$ concentration $(0.5 \%)$, the DF lifetimes $\left(\tau_{\mathrm{DF}}\right)$ of blue bands of DMACDPS singly doped films $(\mathrm{x}=0)$ decreased from 1 to $0.5 \mu$ s while the $\tau_{\mathrm{DF}}$ values of yellow bands decreased to $0.7 \mu$ s (Fig. $3 \mathrm{c}$;
Supplementary Table 2). With a large distance between the dopants, energy transfer depends largely on the intermediate $\mathrm{T}_{1}$ energy levels of the $\mathrm{PO}$ acceptors for exciton migration from blue to yellow dopants.

Apart from the ultrafast intensity rise (6 ns) in yellow bands (insets in Fig. 3c), we recorded appreciable and nearly synchronous rises in both phosphorescent and yellow bands within 100-500 ns. DMAC-DPS with high-lying excited states supports additional energy transfer to the $\mathrm{T}_{1}$ state of the $\mathrm{PO}$ acceptor and then $4 \mathrm{CzTPNBu}$. These synchronous rises further suggest coordination of the exciplex and DMAC-DPS in facilitating energy transfer to $4 \mathrm{CzTPNBu}$. Therefore, upon excitation, a fast, singlet energy transfer occurs, and the triplet energy can be immediately transferred from the exciplexes to DMAC-DPS and then to the $\mathrm{T}_{1}$ energy levels of the PO acceptors within 100-500 ns (Fig. 3d). Almost instantly, the PO acceptors provide the triplet-exciton migration and energy transfer to $4 \mathrm{CzTPNBu}$. As a result, energy harvesting is improved by energy relaying that occurs between blue and yellow dyes, namely $\mathrm{mCP}$ : $\mathrm{PO}$ acceptor $\rightarrow$ DMAC-DPS $\rightarrow \mathrm{PO}$ acceptor $\rightarrow 4 \mathrm{CzTPNBu}$.

WOLED performance. Based on the high $\phi_{\mathrm{PL}}$ values of dualdoped exciplex films and the excellent electrical performance of the PO acceptors and their exciplexes (Supplementary Fig. 3; Supplementary Tables 1 and 2), we further constructed two trilayer TADF-based WOLEDs (W1 and W2) with doubly doped, single-emissive layers (Fig. 4a; Supplementary Fig. 6). Through concentration tuning of $4 \mathrm{CzTPNBu}$, we achieved color modulation from pure white to warm white with a color rendering index beyond 80 and with high chromatic stability (Fig. 4b; Supplementary Figs. 7 and 8; Supplementary Table 3). Commission Internationale de l'Eclairage (CIE) coordinates and correlated color temperatures were $(0.31,0.35) / 6582 \mathrm{~K}$ for the pure white device and $(0.44,0.47) / 3474 \mathrm{~K}$ for the warm white device. Their chromatic purity compared well with a standard daylight source (Illuminant D65) and an incandescent light (Illuminant A).

For the mCP:pDPBITPO-based device W1, we achieved maximum efficiencies of $98.4 \mathrm{~lm} \mathrm{~W}^{-1}$ and $30.6 \%$. $\mathrm{mCP}$ : DpPBITPO-derived W2 generated a power efficiency of $108.2 \mathrm{~lm} \mathrm{~W} \mathrm{~W}^{-1}$, an external quantum efficiency of $32.7 \%$ and a device lifetime of $233 \mathrm{~h}$ at half-initial luminance (Fig. 4c; Supplementary Figs. 9-11). Device degradation is mainly due to photo- and electro-decomposition of DMAC-DPS (Supplementary Figs. 12 and 13). So, using another more stable blue TADF emitter 4,5-bis(carbazol-9-yl)-1,2-dicyanobenzene (2CzPN) instead of DMAC-DPS (Supplementary Fig. 14), device lifetime can be doubled (Supplementary Fig. 11). Based on a $30 \%$ outcoupling ratio of an indium tin oxide glass, we derived $100 \%$ internal quantum efficiencies $\left(\eta_{\mathrm{IQE}}\right)$ for both $\mathrm{W} 1$ and W2. Largesize WOLEDs are hard to fabricate due to stringent requirements on defect control and device homogeneity. We next fabricated TADF WOLED prototypes with lateral dimensions of $8 \mathrm{~cm} \times 8 \mathrm{~cm}$ and efficiencies of $82.7 \mathrm{~lm} \mathrm{~W}^{-1}$ and $25.0 \%$ (Fig. 4d, Supplementary Fig. 15). Our approach is applicable to conventional acceptors. For example, devices W3 and W4, based on two commercial TPBI and BPhen acceptors, achieved maximum efficiencies of $71.3 \mathrm{~lm} \mathrm{~W}^{-1}$ and $22.6 \%$ (Fig. 4c, Supplementary Table 3 ). In addition, our strategy enabled phosphorescent and hyperfluorescent WOLEDs with respective external quantum efficiencies of $32.4 \%$ and $13.7 \%$ (Supplementary Fig. 16).

It is interesting that the maximum $\eta_{\mathrm{EQE}}$ values of $\mathrm{W} 1$ and $\mathrm{W} 2$ are larger than the sum of the corresponding singly doped blue and yellow devices (Supplementary Figs. 17 and 18). We further doped 30\% DMAC-DPS in the emissive layer of the yellow device 

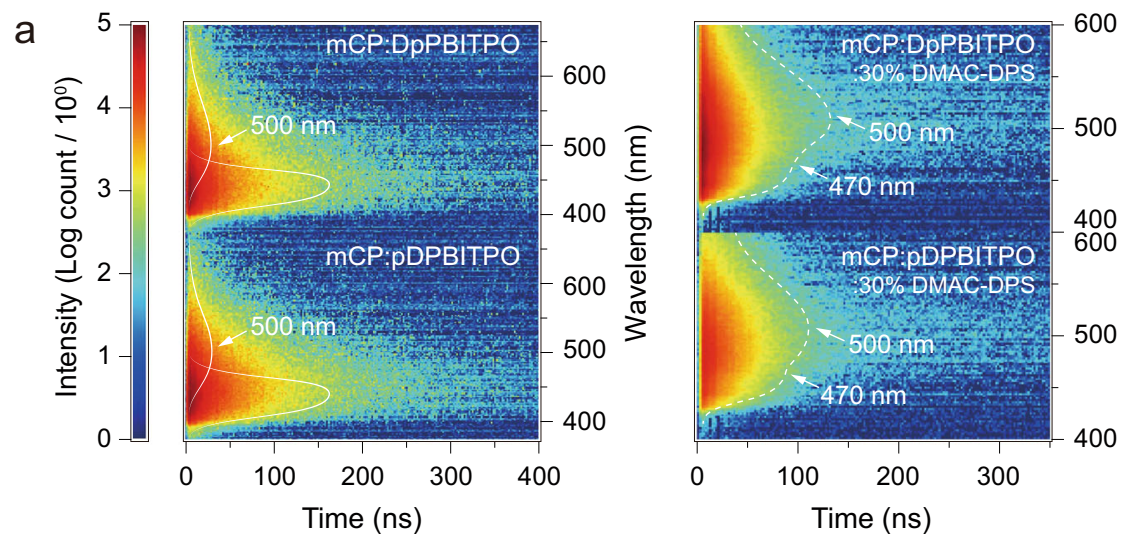

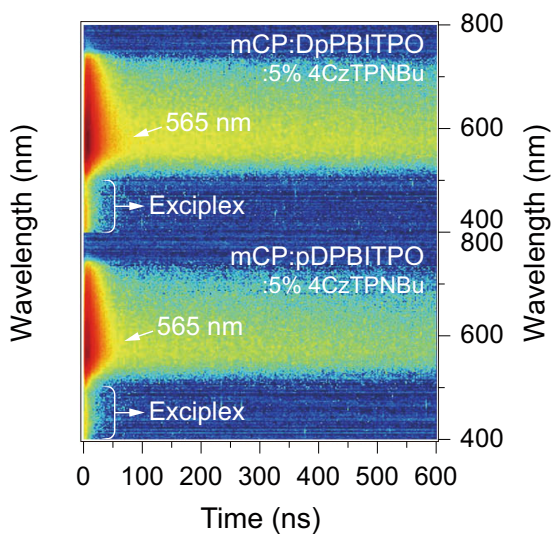

b
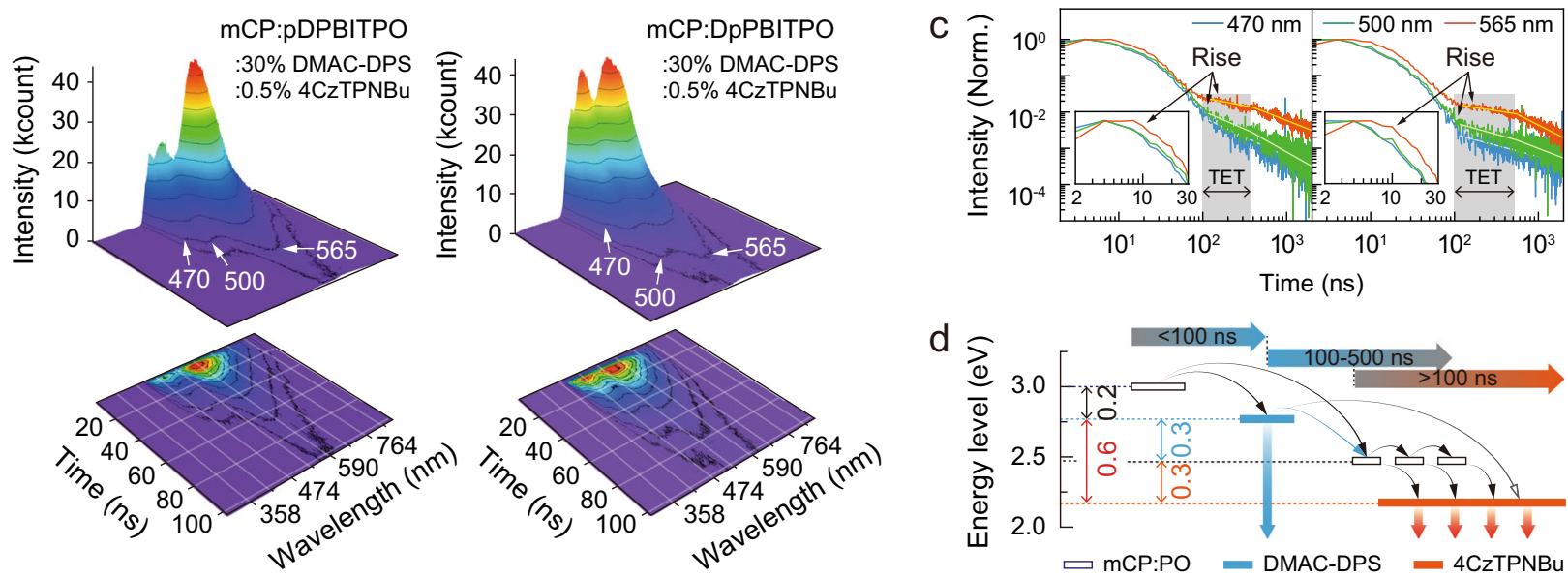

Fig. 3 Transient emission properties of the exciplexes and their TADF dye-doped films. a Time-dependent emission contours of mCP:pDPBITPO and mCP:DpPBITPO films, 30\% DMAC-DPS-doped exciplex films and 4CzTPNBu-doped (5\%) exciplex films, measured in the range of 350-600 nm. b Timedependent emission intensity profiles of mCP:PDPBITPO and mCP:DpPBITPO films doped with 30 wt $\%$ DMAC-DPS and 0.5 wt $\% 4 C z T P N B u$ within 120 ns after excitation. c Time-decay curves of mCP:pDPBITPO (left) and mCP:DpPBITPO (right) films doped with 30 wt.\% DMAC-DPS and 0.5 wt\% 4CzTPNBu. TET refers to a triplet energy transfer from DMAC-DPS to phosphine oxide (PO) acceptors. $\mathbf{d}$ Proposed time-dependent energy transfer processes in dually-doped exciplex films, showing a ladder-like energy relaying enabled by the low-lying $T_{1}$ energy level of the PO acceptor.

(Supplementary Fig. 19). Incorporation of DMAC-DPS dramatically improved the efficiencies by $\sim 40 \%$. Furthermore, we fabricated an mCP:DpPBITPO-based WOLED with dual emissive layers, in which DMAC-DPS and 4CzTPNBu were spatially separated to interrupt triplet energy transfer from DMAC-DPS to DpPBITPO to 4CzTPNBu. This reduced maximum efficiencies to 9.3\%, due to triplet leakage to the $\mathrm{T}_{1}$ levels of DPPBITPO in the blue-emissive layer and nonradiative energy loss in the yellowemissive layer (Supplementary Fig. 20). Taken together, the exciplexes foster energy relaying between the blue and yellow dopants for high-efficiency white electroluminescence.

\section{Discussion}

Our work suggests a general approach to realizing singleemissive-layer TADF WOLEDs with fluorescent lamp efficiency. This strategy, based on exciplex hosts with energy levels simultaneously matching those of blue and yellow dopants, enables a ladder-like, high-efficiency energy relaying. Implementation of these exciplex hosts leads to $100 \%$ exciton harvesting. Furthermore, the realization of purely TADF-based WOLEDs in a simple trilayer device provides a sustainable, cost-effective approach to mitigating fabrication complexity. Ultimately, our molecular design may offer fundamental insight into energy transfer in multicomponent white-emitting systems and add value to the construction of next-generation lighting sources.

\section{Methods}

Preparation of PO acceptors. We designed and synthesized two phosphine oxide acceptors (pDPBITPO and DpPBITPO). These acceptors were fully characterized by ${ }^{1} \mathrm{H}$ NMR, $13 \mathrm{C}$ NMR, $31 \mathrm{P}$ NMR, MS, elemental analysis, and single-crystal (if available) X-ray diffraction analysis (Supplementary Figs 21-26).

General procedure of the cyclization reaction. In an argon atmosphere, aromatic aldehyde $(1 \mathrm{mmol})$ and arylamine $(1 \mathrm{mmol})$ were dissolved in DMF $(5 \mathrm{~mL})$. The resulting solution was heated to $80^{\circ} \mathrm{C}$. A saturated aqueous $\mathrm{Na}_{2} \mathrm{~S}_{2} \mathrm{O}_{5}$ solution $(1 \mathrm{~mL})$ was then added to the mixture and stirred for $20 \mathrm{~h}$ at $80^{\circ} \mathrm{C}$. After cooling to room temperature, the mixture was poured into water. The precipitate was filtered as a crude product and further recrystallized with methanol to afford phenylbenzimidazole derivatives.

2-(4-Bromophenyl)-1-(3,5-dibromophenyl)-1H-benzo[d]imidazole ( $p D P B I T B r)$. pre pared from 4-bromobenzaldehyde and $N$-(3,5-dibromophenyl)benzene-1,2diamine to afford white powder in $73 \%$ yield. ${ }^{1} \mathrm{H}$ NMR (TMS, $\mathrm{CDCl}_{3}, 400$ MHz): $7.880(\mathrm{~d}, J=8.0 \mathrm{~Hz}, 1 \mathrm{H}), 7.809(\mathrm{~m}, 1 \mathrm{H}), 7.532(\mathrm{~d}, J=8.4 \mathrm{~Hz}, 2 \mathrm{H}), 7.454$ $(\mathrm{d}, J=8.8 \mathrm{~Hz}, 4 \mathrm{H}), 7.388(\mathrm{t}, J=7.2 \mathrm{~Hz}, 1 \mathrm{H}), 7.36(\mathrm{t}, J=7.2 \mathrm{~Hz}, 1 \mathrm{H}), 7.259 \mathrm{ppm}$ (d, $J=8.0 \mathrm{~Hz}, 1 \mathrm{H}) ;{ }^{13} \mathrm{C}$ NMR (TMS, $\mathrm{CDCl}_{3}, 100 \mathrm{MHz}$ ): $149.900,141.878$, $137.911,135.705,133.655,130.916,129.751,128.253,127.122,123.642,123.131$, $122.843,122.730,119.244,109.057 \mathrm{ppm}$; HRMS (MALDI-TOF) m/z: $\left[\mathrm{M}+\mathrm{H}^{+}\right]$ 506.875; elemental analysis (\%) for $\mathrm{C}_{19} \mathrm{H}_{11} \mathrm{Br}_{3} \mathrm{~N}_{2}$ : C 45.01, H 2.19, N 5.53; found: C $45.03, \mathrm{H} 2.18, \mathrm{~N} 5.55$.

1-(4-Bromophenyl)-2-(3,5-dibromophenyl)-1H-benzo[d]imidazole (DpPBITBr). pre pared from 3,5-dibromobenzaldehyde and $N$-(4-bromophenyl)benzene-1,2-diamine to afford white powder in $71 \%$ yield. ${ }^{1} \mathrm{H}$ NMR (TMS, $\mathrm{CDCl}_{3}, 400 \mathrm{MHz}$ ): $7.889(\mathrm{~d}, J=7.6 \mathrm{~Hz}, 1 \mathrm{H}), 7.712(\mathrm{~d}, J=8.4 \mathrm{~Hz}, 2 \mathrm{H}), 7.676(\mathrm{t}, J=1.6 \mathrm{~Hz}, 1 \mathrm{H}), 7.644$ $(\mathrm{d}, J=1.6 \mathrm{~Hz}, 2 \mathrm{H}), 7.395(\mathrm{td}, J=7.6 \mathrm{~Hz}, 1.2 \mathrm{~Hz}, 1 \mathrm{H}), 7.337(\mathrm{td}, J=7.6 \mathrm{~Hz}, 1.2 \mathrm{~Hz}$, 1H), 7.244-7.175 ppm (m, 3H); ${ }^{13} \mathrm{C}$ NMR (TMS, $\left.\mathrm{CDCl}_{3}, 100 \mathrm{MHz}\right): 147.946$, 
a

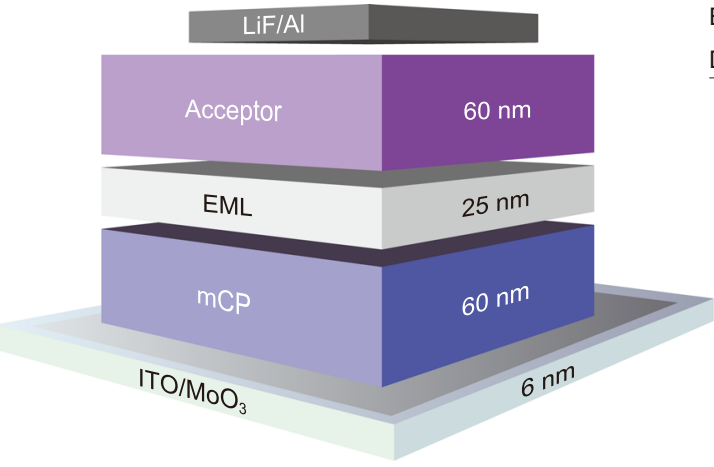

b

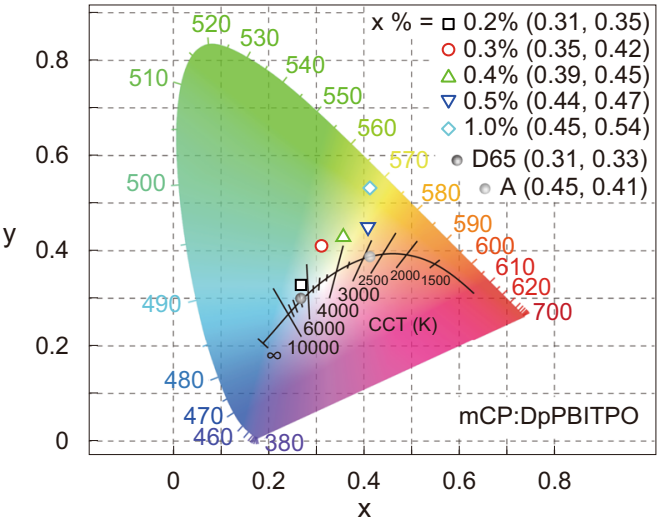

C

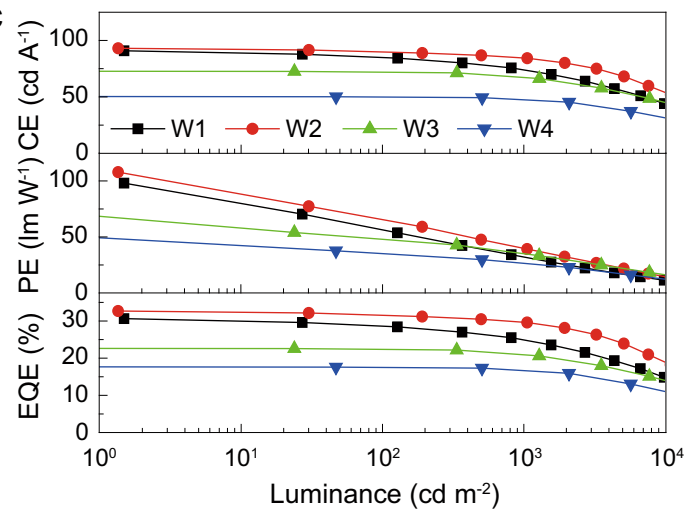

EML: (65-x)\% [mCP:Acceptor]:35\% DMAC-DPS:x\% 4CzTPNBu

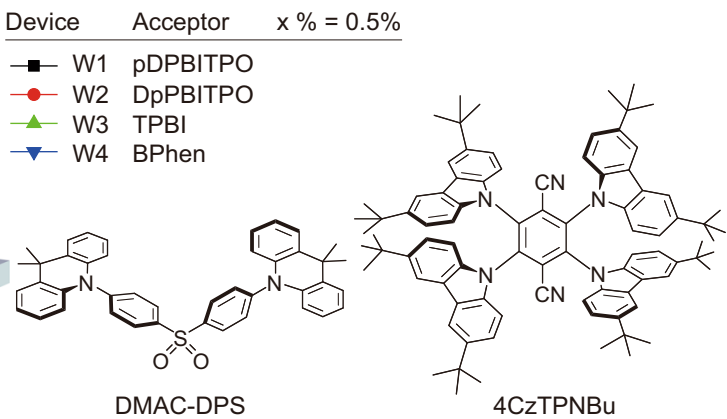

d
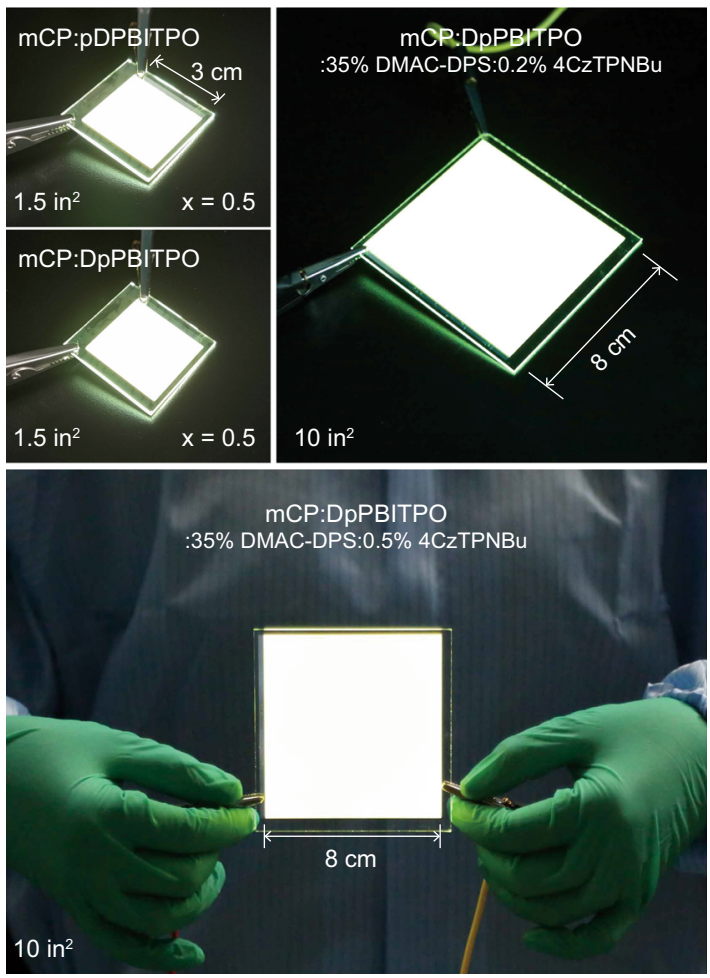

Fig. 4 Electroluminescence characterizations of single-emissive-layer TADF WOLEDs comprising ladder-like energy-relaying exciplexes. a An illustration showing device configuration, the emissive layer components of mCP:pDPBITPO- and mCP:DpPBITPO-hosted white TADF devices, and molecular structures of the doped dye emitters. b Commission International de I'Eclairage (CIE) coordinates of mCP:DpPBITPO-hosted TADF WOLEDs, fabricated with mCP:DpPBITPO:35\% DMAC-DPS:x\%4CzTPNBu. Their chromaticity is comparable to that of a standard daylight source (Illuminant D65) or an incandescent light (Illuminant A). c Efficiency vs. luminance measurements of the devices based on 35\% DMAC-DPS and 0.5\% 4CzTPNBu codoped mCP:pDPBITPO (W1), mCP:DpPBITPO (W2), mCP:TPBI (W3) and mCP:BPhen (W4). d Photographs of mCP:pDPBITPO- and mCP:DpPBITPO-hosted WOLED prototypes with lateral dimensions of $3 \mathrm{~cm} \times 3 \mathrm{~cm}\left(1.5\right.$ inch $\left.^{2}\right)$ and $8 \mathrm{~cm} \times 8 \mathrm{~cm}\left(10\right.$ inch $\left.^{2}\right)$.

$141.754,135.966,134.279,134.031,132.437,131.971,129.887,127.821,123.335$, 122.678, 122.055, 121.942, 119.321, 109.379 ppm; HRMS (MALDI-TOF) m/z: $\left[\mathrm{M}+\mathrm{H}^{+}\right]$506.875; elemental analysis (\%) for $\mathrm{C}_{19} \mathrm{H}_{11} \mathrm{Br}_{3} \mathrm{~N}_{2}$ : C 45.01, $\mathrm{H} 2.19$, N 5.53; found: C 45.02, H 2.19, N 5.56 .

General procedure of phosphorylation. In an argon atmosphere, bromide $(1 \mathrm{mmol}), \mathrm{NaOAc}(3.3 \mathrm{mmol}), \mathrm{Pd}(\mathrm{OAc})_{2}(0.15 \mathrm{mmol})$ and diphenylphosphine $(3.3 \mathrm{mmol})$ were dissolved in DMF $(10 \mathrm{~mL})$. The mixture was heated to $130^{\circ} \mathrm{C}$ and kept at this temperature for $36 \mathrm{~h}$ under stirring. After cooling to room temperature, the reaction was quenched with water $(20 \mathrm{~mL})$. The solution was then extracted with $\mathrm{CH}_{2} \mathrm{Cl}_{2}(3 \times 20 \mathrm{~mL})$. The organic layers were combined and dried with anhydrous sodium sulfate. The solvent was removed in vacuo to afford phosphine precursors. Then, the phosphines were dissolved in $\mathrm{CH}_{2} \mathrm{Cl}_{2}(10 \mathrm{~mL})$ and $\mathrm{H}_{2} \mathrm{O}_{2}$ $(30 \%, 3 \mathrm{~mL})$ was added to the $\mathrm{CH}_{2} \mathrm{Cl}_{2}$ solution dropwise at $0^{\circ} \mathrm{C}$. The mixture was stirred for $2 \mathrm{~h}$, followed by extraction with $\mathrm{CH}_{2} \mathrm{Cl}_{2}(3 \times 10 \mathrm{~mL})$. The organic layers were combined and dried with anhydrous sodium sulfate. The solvent was removed in vacuo, and the residue was purified by flash column chromatography to afford phosphine oxide derivatives.

(5-(2-(4-(Diphenylphosphoryl)phenyl)-1H-benzo[d]imidazol-1-yl)-1,3-phenylene)bis (diphenylphosphine oxide) ( $p D P B I T P O)$. prepared from pDPBITBr to afford white powder in $67 \%$ yield. ${ }^{1} \mathrm{H}$ NMR (TMS, $\left.\mathrm{CDCl}_{3}, 400 \mathrm{MHz}\right)$ : 7.878-7.757 (m, $\left.4 \mathrm{H}\right)$, $7.723\left(\mathrm{dd}, J_{1}=8.0 \mathrm{~Hz}, J_{2}=11.6 \mathrm{~Hz}, 2 \mathrm{H}\right), 7.653\left(\mathrm{dd}, J_{1}=8.0 \mathrm{~Hz}, J_{2}=11.6 \mathrm{~Hz}, 4 \mathrm{H}\right)$, 7.584-7.518 (m, 5H), 7.518-7.418 (m, 15H), 7.418-7.343 (m, 8H), 7.343-7.291 $(\mathrm{m}, 1 \mathrm{H}), 7.258(\mathrm{t}, J=7.6 \mathrm{~Hz}, 1 \mathrm{H}), 7.065 \mathrm{ppm}(\mathrm{d}, J=8.0 \mathrm{~Hz}, 1 \mathrm{H}) ;{ }^{13} \mathrm{C}$ NMR (TMS, $\mathrm{CDCl}_{3}, 100 \mathrm{MHz}$ ): $149.765,141.950,136.643,136.531,136.472,136.338,135.668$, $135.555,135.386,134.038,133.941,133.847,133.802,132.789,132.618,132.535$, $131.878,131.850,131.581,131.274,131.226,131.199,131.178,131.053,130.953$, $130.763,130.711,130.660,130.548,130.200,129.148,128.474,128.355,127.924$, $127.859,127.796,127.754,127.632,123.410,122.848,119.402,109.656 \mathrm{ppm} ;{ }^{31} \mathrm{P}$ NMR (TMS, $\mathrm{CDCl}_{3}, 160 \mathrm{MHz}$ ): 28.195, $27.637 \mathrm{ppm}$; HRMS (MALDI-TOF) m/z: $\left[\mathrm{M}+\mathrm{H}^{+}\right]$871.323; elemental analysis (\%) for $\mathrm{C}_{55} \mathrm{H}_{41} \mathrm{~N}_{2} \mathrm{O}_{3} \mathrm{P}_{3}$ : C 75.86, H 4.75, $\mathrm{N} 3.22$; found: C $75.88, \mathrm{H} 4.76, \mathrm{~N} 3.25$. 
(5-(1-(4-(Diphenylphosphoryl)phenyl)-1H-benzo[d]imidazol-2-yl)-1,3-phenylene)bis (diphenylphosphine oxide) (DpPBITPO). prepared from DpPBITBr to afford white powder in $69 \%$ yield. ${ }^{1} \mathrm{H}$ NMR (TMS, $\mathrm{CDCl}_{3}, 400 \mathrm{MHz}$ ): 8.109 (d, $J=12.4 \mathrm{~Hz}$, $2 \mathrm{H}), 7.901(\mathrm{t}, J=11.2 \mathrm{~Hz}, 1 \mathrm{H}), 7.841-7.744(\mathrm{~m}, 3 \mathrm{H}), 7.730\left(\mathrm{dd}, J_{1}=7.6 \mathrm{~Hz}\right.$, $\left.J_{2}=12.0 \mathrm{~Hz}, 4 \mathrm{H}\right), 7.577(\mathrm{~m}, 2 \mathrm{H}), 7.539-7.430(\mathrm{~m}, 16 \mathrm{H}), 7.421-7.302(\mathrm{~m}, 9 \mathrm{H})$, $7.302-7.241(\mathrm{~m}, 3 \mathrm{H}), 7.191 \mathrm{ppm}(\mathrm{d}, J=8.0 \mathrm{~Hz}, 1 \mathrm{H}) ;{ }^{13} \mathrm{C}$ NMR $\left(\mathrm{TMS}, \mathrm{CDCl}_{3}\right.$, $100 \mathrm{MHz}): 148.973,141.925,138.369,138.339,135.757,135.039,134.937,134.908$, 134.829 , 134.798, 134.261, 134.156, 133.272, 133.187, 133.161, 133.122, 133.057, $132.952,132.264,131.309131 .234,131.146,131.046,130.917,130.866,130.814$, $130.702,130.188,129.656,127.884,127.794,127.762,127.734,127.668,126.195$, $126.072,123.349,122.737,119.345,109.388$ ppm; ${ }^{31} \mathrm{P}$ NMR (TMS, $\mathrm{CDCl}_{3}$ $160 \mathrm{MHz}$ ): 28.437, $27.966 \mathrm{ppm}$; HRMS (MALDI-TOF) m/z: $\left[\mathrm{M}+\mathrm{H}^{+}\right]$871.351; elemental analysis (\%) for $\mathrm{C}_{55} \mathrm{H}_{41} \mathrm{~N}_{2} \mathrm{O}_{3} \mathrm{P}_{3}$ : C 75.86, H 4.75, N 3.22; found: $\mathrm{C} 75.88$, $\mathrm{H} 4.76, \mathrm{~N} 3.25$.

DFT calculations. DFT computations were performed with different parameters for structural optimization and vibrational analysis. The ground state configuration of DpPBITPO was established according to single-crystal data. Ground, singlet, and triplet states in vacuum were optimized by restricted and unrestricted formalisms of Beck's three-parameter hybrid exchange functional ${ }^{32}$ and Lee, and Yang and Parr correlation functional ${ }^{33}$ B3LYP/6-31 G(d,p), respectively. Fully optimized stationary points were further characterized by harmonic vibrational frequency analysis to ensure a real, local minimum without imaginary vibrational frequency. Total energies were also corrected using zero-point energy for both the ground and triplet states. Contours were visualized using Gaussview 5.0. All computations were performed using a Gaussian 09 software package.

Device fabrication and testing. Before loading into a deposition chamber, the ITO substrate was cleaned with detergent and deionized water, dried in an oven at $120^{\circ} \mathrm{C}$ for $4 \mathrm{~h}$, and treated with oxygen plasma for $3 \mathrm{~min}$. Devices were fabricated by evaporating organic layers at a rate of $0.1-0.2 \mathrm{~nm} \mathrm{~s}^{-1}$ onto the ITO substrate sequentially at a pressure below $4 \times 10^{-4} \mathrm{~Pa}$. Onto the electron-transporting layer, a layer of LiF with $1-\mathrm{nm}$ thickness was deposited at a rate of $0.1 \mathrm{~nm} \mathrm{~s}^{-1}$ to improve electron injection. Finally, a $100-\mathrm{nm}$ layer of $\mathrm{Al}$ was deposited at a rate of $0.6 \mathrm{~nm} \mathrm{~s}^{-1}$ as the cathode. The emission area of the devices was $0.09 \mathrm{~cm}^{2}$, as determined by the overlapped area of the anode and the cathode. After fabrication, devices were immediately transferred to a glove box for encapsulation with glass coverslips using epoxy glue. EL spectra and CIE coordinates were measured using a PR655 spectrum colorimeter. Current-density-voltage and brightness-voltage curves of the devices were measured using a Keithley 4200 source meter and a calibrated silicon photodiode. All measurements were carried out at room temperature under ambient conditions. For each structure, four devices were fabricated in parallel to confirm performance repeatability. The data reported herein were those closest to the average results.

Photophysical measurement. Steady-state emission spectra were measured using an Edinburgh FPLS 920 fluorescence spectrophotometer. TADF dye-doped films $(100 \mathrm{~nm})$ were prepared by vacuum evaporation for optical analysis. Photoluminescence quantum yields (PLQY, $\phi_{\mathrm{PL}}$ ) of these films were measured through a Labsphere 1-M-2 $(\phi=6 ")$ integrating sphere coated with Benflect having efficient light reflection from 200 to $1600 \mathrm{~nm}$, which was integrated with FPLS 920. The absolute $\phi_{\mathrm{PL}}$ determination of the sample was performed with two spectral (emission) scans, with the emission monochromator scanning over the Rayleigh scattered light from the sample and a blank substrate. The first spectrum recorded the scattered light and the sample emission, and the second spectrum recorded the scattered light of the Benflect coating. Integration and subtraction of the scattered light in the two spectra are equal to the number of photons absorbed by the samples $\left(\mathrm{N}_{\mathrm{a}}\right)$, while integration of the sample emission is equal to the number of photons emitted $\left(\mathrm{N}_{\mathrm{e}}\right)$. Then, absolute $\phi_{\mathrm{PL}}$ can be estimated according to the equation of $\phi_{\mathrm{PL}}=\mathrm{N}_{\mathrm{e}} / \mathrm{N}_{\mathrm{a}}$. Spectral correction (emission arm) was applied to raw data after background subtraction, and from these spectrally corrected curves, the quantum yield was calculated using an F900 software wizard.

Time-resolved photoluminescence measurements with nanosecond time resolution were acquired with a gated, intensified CCD camera system (Andor iStar DH740 CCI-010) connected to a grating spectrometer (Andor SR303i) at room temperature. Excitation (wavelength: $350 \mathrm{~nm}$ ) was performed with femtosecond laser pulses, generated by introducing $1 \mathrm{kHz}$ pulses (pulse length: $80 \mathrm{fs}$ ) from a central Ti:sapphire amplifier system (Spectra-Physics Solstice) to a TOPAS optical parametric amplifier (Light Conversion). Time-resolved photoluminescence of the sample was obtained by stepping the intensified CCD gate delay relative to the pump pulse. The gate width was $2 \mathrm{~ns}$.

\section{Data availability}

Data that support the findings of this study are available from the corresponding authors upon request. The X-ray crystallographic coordinates for the DpPBITPO structure reported in this study have been deposited at the Cambridge Crystallographic Data Centre (CCDC), under deposition numbers 2077630. These data can be obtained free of charge from The Cambridge Crystallographic Data Centre via www.ccdc.cam.ac.uk/ data_request/cif.
Received: 5 March 2021; Accepted: 14 May 2021; Published online: 15 June 2021

\section{References}

1. Kido, J., Kimura, M. \& Nagai, K. Multilayer white light-emitting organic electroluminescent device. Science 267, 1332-1334 (1995).

2. Sun, Y. R. et al. Management of singlet and triplet excitons for efficient white organic light-emitting devices. Nature 440, 908-912 (2006).

3. Reineke, S. et al. White organic light-emitting diodes with fluorescent tube efficiency. Nature 459, 234-238 (2009).

4. Uoyama, H., Goushi, K., Shizu, K., Nomura, H. \& Adachi, C. Highly efficient organic light-emitting diodes from delayed fluorescence. Nature 492, 234-238 (2012).

5. Tao, Y. et al. Thermally activated delayed fluorescence materials towards the breakthrough of organoelectronics. Adv. Mater. 26, 7931-7958 (2014).

6. Ahn, D. H. et al. Highly efficient blue thermally activated delayed fluorescence emitters based on symmetrical and rigid oxygen-bridged boron acceptors. Nat. Photon 13, 540-546 (2019).

7. D'Andrade, B. W. \& Forrest, S. R. White organic light-emitting devices for solid-state lighting. Adv. Mater. 16, 1585-1595 (2004).

8. Kamtekar, K. T., Monkman, A. P. \& Bryce, M. Recent advances in white organic light-emitting materials and devices (WOLEDs). Adv. Mater. 22, 572-584 (2010).

9. Yu, L. et al. Red, green, and blue light-emitting polyfluorenes containing a dibenzothiophene-S,S-dioxide unit and efficient high-color-rendering-index white-light-emitting diodes made therefrom. Adv. Funct. Mater. 23, 4366-4376 (2013)

10. Wang, Q. \& Ma, D. Management of charges and excitons for highperformance white organic light-emitting diodes. Chem. Soc. Rev. 39, 2387-2398 (2010).

11. Zhao, F. et al. Spatial exciton allocation strategy with reduced energy loss for high-efficiency fluorescent/phosphorescent hybrid white organic lightemitting diodes. Mater. Horiz. 4, 641-648 (2017).

12. Cui, L. S. et al. Controlling synergistic oxidation processes for efficient and stable blue thermally activated delayed fluorescence devices. Adv. Mater. 28, 7620-7625 (2016).

13. Jankus, V. et al. Highly efficient TADF OLEDs: how the emitter-host interaction controls both the excited state species and electrical properties of the devices to achieve near $100 \%$ triplet harvesting and high efficiency. Adv. Funct. Mater. 24, 6178-6186 (2014).

14. Han, C., Zhang, Z., Ding, D. \& Xu, H. Dipole-dipole interaction management for efficient blue thermally activated delayed fluorescence diodes. Chemistry 4 , 2154-2167 (2018).

15. Fan, C. et al. Dibenzothiophene-based phosphine oxide host and electrontransporting materials for efficient blue thermally activated delayed fluorescence diodes through compatibility optimization. Chem. Mater. 27, 5131-5140 (2015).

16. Liu, Y., Li, C., Ren, Z., Yan, S. \& Bryce, M. R. All-organic thermally activated delayed fluorescence materials for organic light-emitting diodes. Nat. Rev. Mater. 3, 18020 (2018).

17. $\mathrm{Wu}, \mathrm{Z}$. et al. Strategic-tuning of radiative excitons for efficient and stable fluorescent white organic light-emitting diodes. Nat. Commun. 10, 2380 (2019).

18. Li, Y. et al. Design strategy of blue and yellow thermally activated delayed fluorescence emitters and their all-fluorescence white OLEDs with external quantum efficiency beyond 20\%. Adv. Funct. Mater. 26, 6904-6912 (2016).

19. Zhang, B. et al. High-efficiency single emissive layer white organic lightemitting diodes based on solution-processed dendritic host and new orangeemitting iridium complex. Adv. Mater. 24, 1873-1877 (2012).

20. Zhang, Q. et al. Efficient blue organic light-emitting diodes employing thermally activated delayed fluorescence. Nat. Photon. 8, 326-332 (2014).

21. Förster, T. Zwischenmolekulare energiewanderung und fluoreszenz. Ann. Phys. (Berl.) 437, 55-75 (1948).

22. Dexter, D. L. A theory of sensitized luminescence in solids. J. Chem. Phys. 21, 836-850 (1953).

23. $\mathrm{Xu}, \mathrm{H}$. \& Huang, W. Spectroscopic study of intramolecular energy transfer in a phosphine oxide $\mathrm{Eu}^{3+}$ complex: a stepwise process induced by intermediate energy levels. J. Photochem. Photobiol. A 217, 213-218 (2011).

24. Sun, J. et al. Charge-transfer exciton manipulation based on hydrogen bond for efficient white thermally activated delayed fluorescence. Adv. Funct. Mater. 30, 1908568 (2020).

25. Ding, D. et al. Highly efficient and color-stable thermally activated delayed fluorescence white light-emitting diodes featured with single-doped single emissive layers. Adv. Mater. 32, 1906950 (2020).

26. Feng, J. \& Zhang, H. Hybrid materials based on lanthanide organic complexes: a review. Chem. Soc. Rev. 42, 387-410 (2013). 
27. Wang, Z. et al. The application of charge transfer host based exciplex and thermally activated delayed fluorescence materials in organic light-emitting diodes. Org. Electron. 66, 227-241 (2019).

28. Duan, C., Han, C., Du, R., Wei, Y. \& Xu, H. High-efficiency blue dual-emissive exciplex boosts full-radiative white electroluminescence. Adv. Opt. Mater. 6 1800437 (2018).

29. Liu, X.-K. et al. Prediction and design of efficient exciplex emitters for highefficiency, thermally activated delayed-fluorescence organic light-emitting diodes. Adv. Mater. 27, 2378-2383 (2015).

30. Gibson, J., Monkman, A. P. \& Penfold, T. J. The importance of vibronic coupling for efficient reverse intersystem crossing in thermally activated delayed fluorescence molecules. ChemPhysChem 17, 2956-2961 (2016).

31. Chaudhuri, D. et al. Metal-free OLED triplet emitters by side-stepping Kasha's rule. Angew. Chem. Int. Ed. 52, 13449-13452 (2013).

32. Becke, A. D. Density-functional thermochemistry. III. The role of exact exchange. J. Chem. Phys. 98, 5648-5652 (1993).

33. Lee, C., Yang, W. \& Parr, R. G. Development of the Colle-Salvetti correlationenergy formula into a functional of the electron density. Phys. Rev. B 37, 785-789 (1988).

\section{Acknowledgements}

This project was financially supported by the Changjiang Scholar Program of the Chinese Ministry of Education (Q2016208), the National Natural Science Foundation of China (92061205, U1801258, 51873056 and 22005088), the National Basic Research Program of China (973 Program, 2015CB932200), and National Research Foundation, Prime Minister's Office, Singapore under its Investigatorship Award Program (NRF-NRFI05-2019-0003).

\section{Author contributions}

C.H. and R.D. contributed equally to this work. W.H., X.L., and H.X. conceived the projects. C.H., R.D., S.H., P.M., J.B., M.S., C.D., and Y.W. performed the experiments C.H., R.D., S.H., H.X., X.L., and W.H. analyzed the data and wrote the paper. All authors commented on the manuscript.

\section{Competing interests}

The authors declare no competing interests.

\section{Additional information}

Supplementary information The online version contains supplementary material available at https://doi.org/10.1038/s41467-021-23941-z.

Correspondence and requests for materials should be addressed to H.X., X.L. or W.H

Peer review information Nature Communications thanks Sebastian Reineke and the other, anonymous, reviewer(s) for their contribution to the peer review of this work.

Reprints and permission information is available at http://www.nature.com/reprint

Publisher's note Springer Nature remains neutral with regard to jurisdictional claims in published maps and institutional affiliations.
Open Access This article is licensed under a Creative Commons Attribution 4.0 International License, which permits use, sharing, adaptation, distribution and reproduction in any medium or format, as long as you give appropriate credit to the original author(s) and the source, provide a link to the Creative Commons license, and indicate if changes were made. The images or other third party material in this article are included in the article's Creative Commons license, unless indicated otherwise in a credit line to the material. If material is not included in the article's Creative Commons license and your intended use is not permitted by statutory regulation or exceeds the permitted use, you will need to obtain permission directly from the copyright holder. To view a copy of this license, visit http://creativecommons.org/ licenses/by/4.0/

(C) The Author(s) 2021 